\title{
Téoros
}

Revue de recherche en tourisme

\section{Les rôles respectifs des structures organisationnelles du tourisme dans les pays en transition}

\section{L'exemple de l'Allbanie}

\section{Monika Peppo}

Volume 19, numéro 3, automne 2000

Organisations touristiques en mutation

URI : https://id.erudit.org/iderudit/1071741ar

DOI : https://doi.org/10.7202/1071741ar

Aller au sommaire du numéro

Éditeur(s)

Université du Québec à Montréal

ISSN

0712-8657 (imprimé)

1923-2705 (numérique)

Découvrir la revue

Citer cet article

Peppo, M. (2000). Les rôles respectifs des structures organisationnelles du tourisme dans les pays en transition : l'exemple de l'Albanie. Téoros, 19(3), 36-39. https://doi.org/10.7202/1071741ar d'utilisation que vous pouvez consulter en ligne. 


\section{LES RÔLES RESPECTIFS DES STRUCTURES ORGANISATIONNELLES DU TOURISME DANS LES PAYS EN TRANSITION L'EXEMPLE DE L'AlBANIE}

\section{Monika Peppo}

La gestion du développement du tourisme peut reposer sur des structures publiques et privées de différents niveaux : régional, national, international et urbain, mais aussi de la communauté de la zone touristique.

Les structures organisationnelles efficaces jouent un rôle essentiel dans la planification, le développement, le marketing, la coordination et le management du tourisme d'un pays et d'une région. Néanmoins, on doit adapter les structures du secteur public et gouvernemental, ainsi que leur niveau d'intervention dans le domaine touristique, aux besoins spécifiques et à la structure idéologique et politique du pays ainsi qu'à la stratégie de développement touristique. Ce sont les décisions politiques qui définissent jusqu'où le gouvernement intervient dans le développement du tourisme. Une des bases de cette théorie est que le gouvernement - national, régional ou une combinaison des deux paliers de gouvernements - doit avoir la responsabilité de la gestion touristique, de la planification, du développement, de la standardisation des services et des équipements touristiques, de quelques aspects de la promotion et, surtout, de la qualité environnementale de la région. Autrement, le tourisme ne sera pas intégré, planifié ni contrôlé et il ne pourra pas répondre aux besoins de la région et de sa communauté.

\section{LES DIFFÉRENTS NIVEAUX DES STRUCTURES ORGANI- SATIONNELLES EN ALBANIE}

De nombreuses organisations jouent un rôle important dans le développement du tourisme en Albanie. Parmi celles-ci, notons :
- au plan international - l'OMT (Organisation mondiale du tourisme), l'IATA (International Air Transport Association), l'OCDE (Organisation de coopération et de développement économiques), etc. ;

- au plan national - le Comité du développement du tourisme, Albturism, les organisations non gouvernementales, les syndicats et les confédérations ;

- au plan régional - les bureaux de promotion et de relations étrangères auprès de la préfecture, du conseil régional et de la mairie, les chambres de commerce et d'industrie, les conseils de l'aménagement du territoire de la ville et de la région, les agences de développement régional, ainsi que plusieurs organisations privées nationales et régionales.

La participation de l'État et le rôle des organisations publiques et privées diffèrent selon le système politique, la stratégie mise en œuvre et le niveau de développement du pays.

\section{LES CHANGEMENTS DES STRUCTURES ORGANI- SATIONNELLES : D'UNE ÉCONOMIE PLANIFIÉE À UNE ÉCONOMIE DE MARCHÉ}

En 1992, le changement de système politique et économique en Albanie a amené

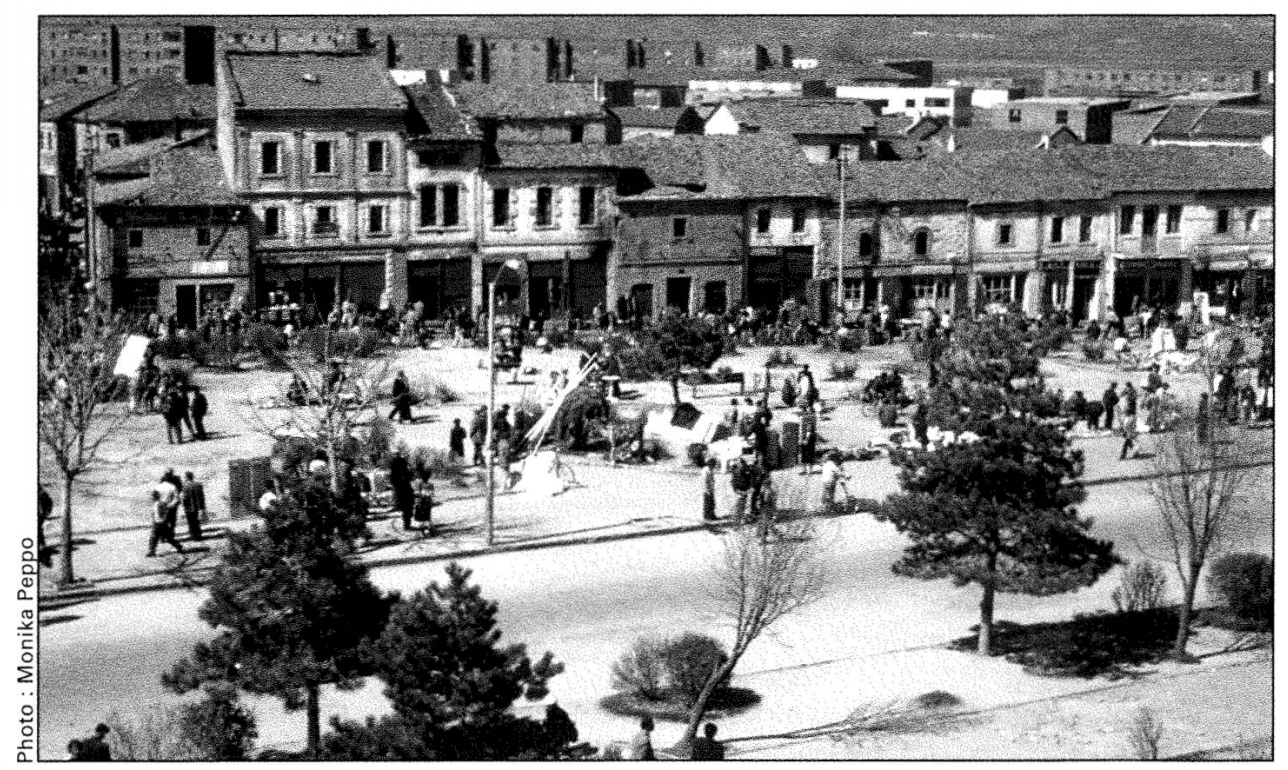

Vue de Korça 
un bouleversement des structures organisationnelles de l'économie et de la société. Le tourisme, en tant que phénomène économique et social, n'y a pas échappé. Les structures de base sur lesquelles s'appuyait le tourisme albanais pendant la période du socialisme étaient très centralisées et directement dépendantes de l'État. Albturism était l'entreprise centrale qui gérait les structures les plus importantes d'hébergement, de restauration, de loisirs et de commerce touristique. Le régime communiste n'avait jamais considéré le tourisme comme une industrie. Il n'y avait aucune stratégie de développement et, à la différence des autres secteurs de l'économie, le tourisme n'était jamais cité dans les plans quinquennaux.

Le tourisme interne jouait un rôle important par comparaison au tourisme international, jugé « dangereux »pour une Albanie fermée. Les rares touristes étrangers venaient toujours en groupes et étaient très contrôlés : l'Albturism organisait les séjours et les visites. L'Albanie touristique était très limitée dans le sens physique, car plusieurs sites touristiques étaient « interdits »au public pour différentes raisons politiques, stratégiques et idéologiques. Comme tous les autres secteurs de l'économie et peut-être même plus que tous les autres, le secteur touristique était très idéologique. Il fallait présenter une Albanie belle et sans problème aux yeux des étrangers. De ce fait, le tourisme était un secteur sensible étroitement lié à l'État.

L'Albanie d'aujourd' hui, ouverte et démocratique, a commencé à penser au tourisme, à cette «barque de Noé », mais sans réellement s'appuyer sur une stratégie. Le premier gouvernement démocratique a vu, dans cette industrie, une source de devises étrangères et un catalyseur budgétaire. Conscients ou non de la priorité à accorder au tourisme, les chefs du Gouvernement démocratique ont décidé de créer tout d'abord la structure la plus haute : le ministère du Tourisme. Un tel ministère est justifié quand le tourisme est déjà - ou envisage d'être - un des secteurs les plus importants de l'économie. Il donne à ce secteur une image publique et un statut politique plus forts. Or, la réalité s'est révélée bien différente de la théorie. Le budget que l'État a accordé à ce ministère ne couvrait que son administration et son fonctionnement. Selon la stratégie du développement du tourisme 1993-2010, le Ministère prévoyait en 1993 un budget de promotion de 150000 à 200000 \$ US ! Le

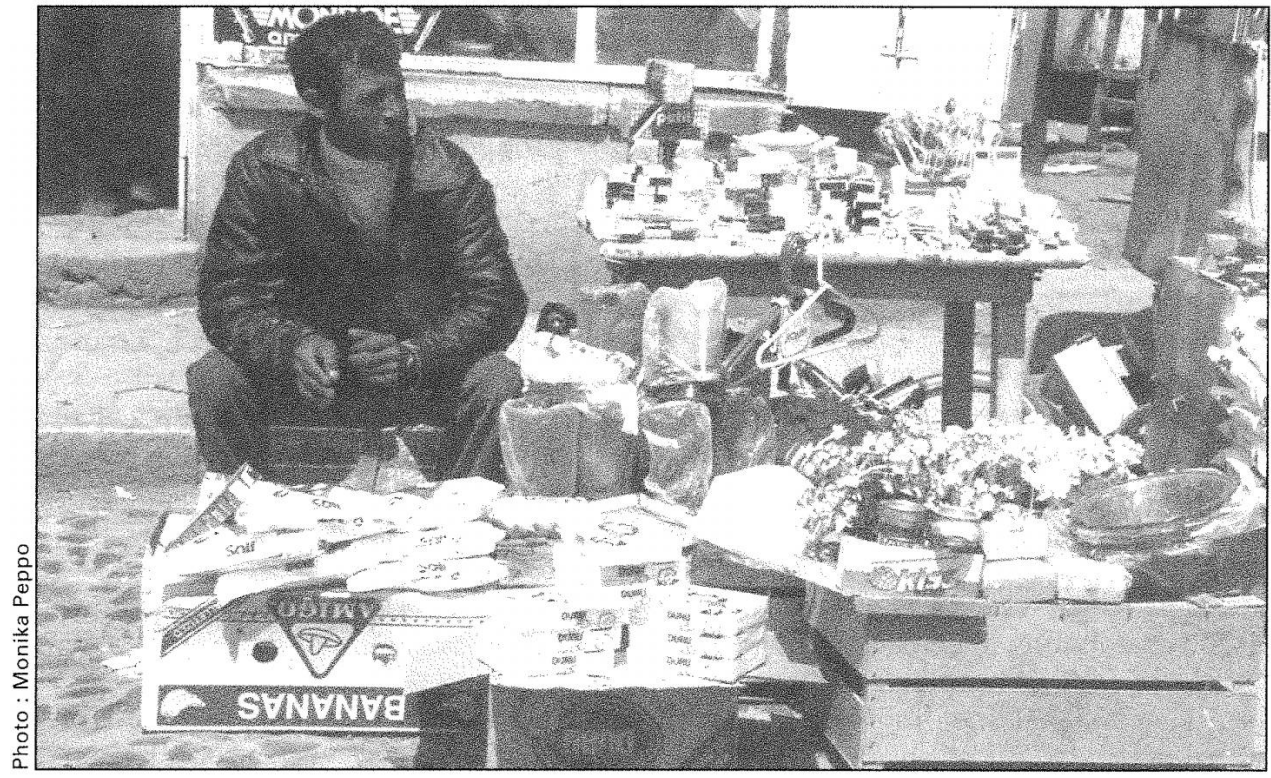

Marchand de rue à Korça

ministère du Tourisme comptait beaucoup sur l'aide financière des organismes internationaux, surtout en ce qui concerne le développement des infrastructures; les investisseurs étrangers et albanais devaient financer la superstructure touristique. Cette stratégie n'a jamais été mise en œuvre et les fonds n'ont jamais été octroyés. Plusieurs facteurs expliquent ce dysfonctionnement : tout d'abord, l'Albanie n'était pas prête à atteindre cet objectif ambitieux, par manque quasi total de structures organisationnelles touristiques de haut niveau ; ensuite, aucune institution n'avait formé, jusqu'à ce jour, des cadres et des spécialistes en tourisme ; enfin, le fonctionnement du ministère du Tourisme nécessitait également une coopération très forte avec les autres ministères. Malheureusement il s'est trouvé seul, personne n'ayant compris que le tourisme est une activité complexe et multisectorielle qui exige une coordination de toutes les structures organisationnelles gouvernementales et privées. Dans les pays sous-développés avec un faible secteur privé, le gouvernement doit jouer un rôle d'autant plus fort pour développer le tourisme. Il doit prendre en charge le marketing et l'investissement et il doit favoriser les initiatives visant à encourager le développement du tourisme.

$\mathrm{Vu}$ le mauvais fonctionnement du ministère du Tourisme, le gouvernement démocratique (qui a gagné encore une fois les élections) a décidé de créer un ministère commun au tourisme et aux transports. En 1996, le tourisme en Albanie était donc géré par ce ministère du Tourisme et des Transports. Mais, une question difficile se pose quand le tourisme est associé à d'autres fonctions dans un ministère mixte : quelle sera la synergie optimale pour atteindre le développement du tourisme et sa gestion effective ?

En 1997, le pouvoir est passé aux socialistes qui ont changé le schéma organisationnel du gouvernement. Le tourisme cette fois est passé au ministère de l'Économie et du Développement. Selon l'opinion des spécialistes, les problèmes du tourisme sont restés « orphelins »et personne ne les a pris en charge. Après beaucoup d'efforts, on a créé un Comité de développement du tourisme, entité légale autonome responsable des différents aspects du tourisme en Albanie.

\section{LES STRUCTURES LOCALES ET RÉGIONALES}

Le Bureau de promotion et de relations étrangères à la mairie de Korça (ville du sud de l'Albanie), en collaboration avec des organismes étrangers (les pays de la Communauté européenne et les pays voisins), a élaboré des projets de développement de la région, notamment de développement touristique. C'est la Communauté européenne qui finance la plupart de ces projets qui ne dépassent pas le stade de la promotion ou de l'étude de cas. On peut citer ici le programme PHARE-CROSS BORDER qui compte plusieurs projets, dont « L'étude et l'amélioration du vieux bazar de Korça », comme un point fort du potentiel touristique. Un autre programme, 
PHARE-CREDO EAST-EAST CROSS BORDER COOPERATION, vise le développement du tourisme entre Korça (Alb) et Ohrid (Macédoine). Le rôle des organismes étrangers est plus fort que celui des organismes autochtones, mais il faut souligner le rôle et la participation de plus en plus importants des organismes non gouvernementaux à la vie quotidienne et au développement de la région. Les plus actifs sont des organismes intéressés aux questions d'environnement qui ont amené des projets ambitieux et très utiles au développement du tourisme. En effet, la stratégie nationale du développement du tourisme souligne l'importance du tourisme écologique en Albanie, le considérant comme l'une des formes les plus pertinentes de tourisme. On peut mentionner ici l'organisation Perla qui a entrepris jusqu'à maintenant plusieurs projets pour protéger et améliorer l'environnement et surtout les sites naturels touristiques de la région. Par ailleurs, de tels organismes mènent de temps en temps des campagnes de sensibilisation visant un environnement durable, base d'un tourisme durable.

La Chambre de commerce et d'industrie de Korça joue un rôle de coordonnateur entre, d'une part, les différents niveaux de pouvoir et, d'autre part, les secteurs public et privé. La Chambre a organisé des groupes de travail en vue de préparer un guide touristique de Korça. Voilà un bon exemple de coopération entre spécialistes d'organismes publics et privés. Un autre organisme public - financé et géré par des fonds étrangers -, l'Agence régionale du développement, a réalisé des études sur les potentiels de la région, dont les potentiels touristiques.

L'Université de Korça et en particulier le Département du tourisme, en collaboration avec l'ESTHUA (Université d'Angers), ont été les premiers à comprendre l'importance du secteur touristique. Les cadres formés en Europe de l'Ouest ont transmis leurs connaissances et participent activement à tous les projets de développement du tourisme entrepris par les structures organisationnelles publiques et privées de la région et même audelà. Le pouvoir local et les autres organismes publics et privés sont déjà conscients du rôle irremplaçable du Département du tourisme dans ce développement. Les professeurs et les étudiants font partie de groupes de travail des différents projets. Ajoutons que cette collaboration a mis du temps à se mettre en place.

Le Conseil d'aménagement du territoire, au niveau de la ville et de la région, est un organisme public qui dispose d'un grand pouvoir, plus particulièrement pendant la période de transition. C'est ce Conseil qui est chargé de faire respecter la loi pour tout ce qui concerne la préservation de l'originalité et des valeurs historiques et culturelles des sites de la ville et de la région. C'est encore ce Conseil qui accorde maintenant les permis de construire et qui veille à mettre en œuvre au niveau national les directives gouvernementales, ce qui ne se fait pas sans difficulté. En effet, l'Albanie, pays en développement, a attribué davantage de responsabilités et d'activités au pouvoir (soit central, soit local). Le rôle des organismes publics gouvernementaux dans la période de transition est vital surtout pour des problèmes assez délicats comme le développement du tourisme qui nécessite une coopération et une coordination de tous les acteurs. Le secteur privé s'engage de plus en plus, mais agit de façon isolée, spontanée. Son rôle se limite à la construction de quelques structures hôtelières et de restauration le

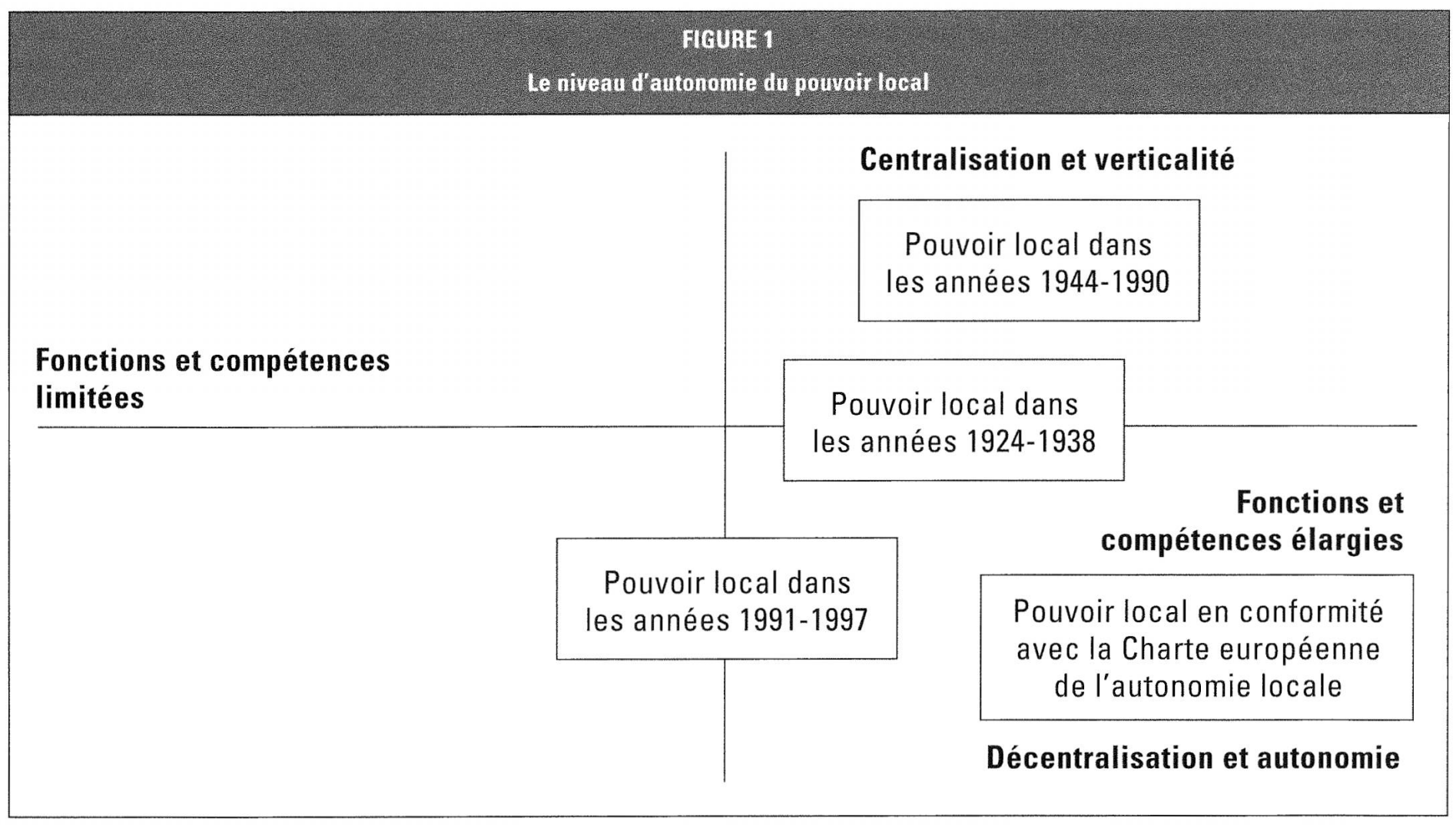


long de la côte albanaise et dans les grandes villes. Dans un pays assez pauvre comme l'Albanie, il paraît impossible au secteur privé d'entreprendre des projets ambitieux en tourisme.

\section{LES PROBLÈMES DE DÉCENTRALISATION}

Les bureaux de promotion et de relations étrangères ont été créés au niveau régional auprès des préfectures, des conseils régionaux et des mairies. Ces bureaux ne sont pas spécialisés en tourisme, mais s'impliquent dans les projets de développement de la région, notamment les projets de développement du tourisme. Les structures régionales sont toujours restées détachées des structures nationales. Les relations entre les deux pouvoirs, central et régional, restent encore froides. L'Albanie est sortie d'un système hyper centralisé, où les initiatives étaient orientées et approuvées par le pouvoir central ; elle n'a aucune tradition en matière de décentralisation. Aussi, même si elles sont encore limitées, les fonctions et les compétences du pouvoir local apparaissent importantes par rapport aux périodes précédentes.

Pendant la période du socialisme, les grandes fonctions économiques et sociales, réalisées par les structures locales, faisaient partie de la division verticale des devoirs et des compétences dans un État centralisé. Leur budget était complètement couvert par le budget de l'Etat, avec une gestion très rigoureuse des dépenses. En 1992, le Parlement démocratique a approuvé la Loi du pouvoir local où, pour la première fois, on reconnaissait l'autonomie politique, administrative et économique du pouvoir local. Cette Loi a crée deux niveaux de pouvoir, indépendants l'un de 1'autre, établissant les prémisses de l'élargissement, dans un futur très proche, des compétences et des fonctions des organisations locales. Les élections au niveau local ont été gagnées par l'opposition, ce qui a freiné la politique du pouvoir central (tenu par les démocrates) à la poursuite de la réforme du pouvoir local.

Il en résulte des tendances claires de centralisation dans les différents secteurs de l'économie, de l'éducation et de la santé. Les revenus des organismes locaux dépendent à $95 \%$ du budget de l'État et la structure des dépenses reste toujours rigoureu-

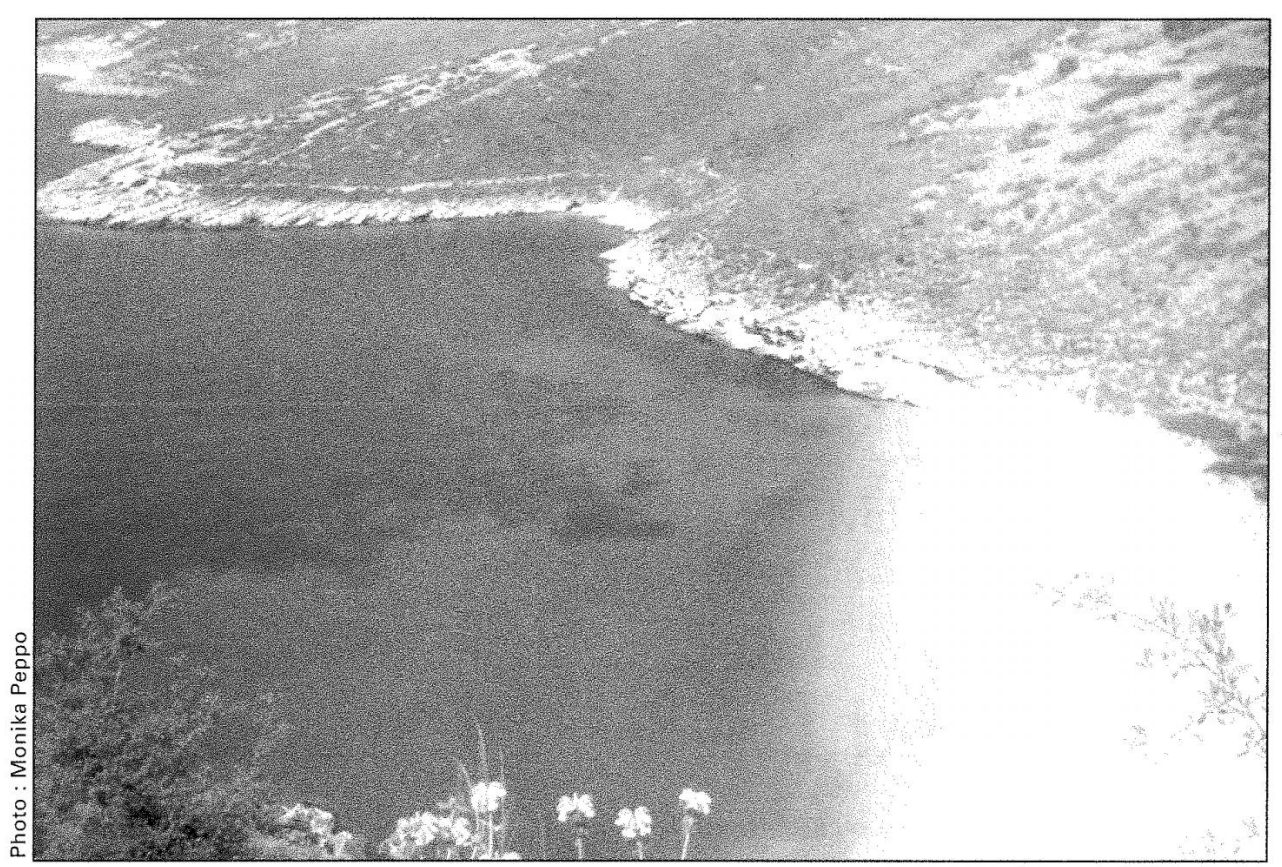

Littoral au sud de l'Albanie : mer Ionienne

sement contrôlée par le ministère des Finances. En raison des problèmes macroéconomiques liés à la période de transition, de l'état de l'économie, mais également de l'instabilité politique, le pouvoir local reste dans l'ombre et ne peut pas jouer de rôle important dans le développement du tourisme. Il met en cuvre la politique définie par le pouvoir central.

L'esprit de gouvernement au niveau local est encore faible en raison d'une longue expérience totalitaire de programmation et d'exécution profondément centralisées. Dans la région de Korça, les organisations publiques locales font beaucoup d'efforts pour prendre l'initiative du développement touristique, mais elles ne peuvent pas intervenir sur le plan macro-économique ni dépasser les frontières des zones prioritaires définies par la politique centrale du tourisme. Pendant la période d'économie planifiée, il n'y avait pas de contradiction entre les stratégies élaborées par le gouvernement central et leur application à la base. Aujourd'hui, il existe de nombreuses contraintes à un progrès normal. Les propriétaires privés et les structures organisationnelles privées jouent maintenant un rôle important dans la société. Le manque d'expérience de collaboration entre les structures publiques et privées amène parfois des problèmes sérieux. Les structures publiques doivent être conscientes de leur nouveau rôle dans une économie de marché.
Monika Peppo a été chargée d'enseignement à l'ESTHUA (École supérieure de tourisme et d'hôtellerie de l'Université d'Angers); elle est actuellement étudiante au doctorat - programme de co-tutelle entre l'Université d'Angers et l'Université de Korça en Albanie.

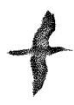

\section{BIBLIOGRAPHIES}

Centre de documentation de IUP-ESTHUA, Évaluation et mise en valeur du potentiel touristique de la région de Korça, Angers, France.

Harrison, Lynn C., and Winston Carlyle Husbands (1996), Practicing Responsible Tourism: International Case Studies in Tourism Planning Policy and Development, New York: J.Wiley.

Inskeep, Edward (1991), Tourism Planning: An Integrated and Sustainable Development Approach, New York: Van Nostrand Reinhold.

Ministère du Tourisme de l'Albanie (1993), La stratégie de développement du tourisme 19932010, Tirana, Albanie.

Peppo, Monika (1998), Le rôle du pouvoir central et local au développement du tourisme, Mémoire de diplôme, Département de Tourisme, Université de Korça, Albanie.

Peppo, Monika (1993), Mémoire de stage, E.S.T.H.U.A., Université d'Angers.

Thomo, Pirro (1988), Korça; urbanistika dhe arkitektura (Korça; L'urbanisation et l'architecture), Tirana, Albanie.

UNDP (1998), Raporti $i$ zhvillimit njerezor ne Shqiperi (Le rapport du développement humain en Albanie), Tirana, Albanie. 